Voix et Images

volxetimages

\title{
Critique théâtralisée des esthétiques marchandes
}

Les dramaturgies performatives d'Olivier Choinière et de

Guillaume Corbeil

\section{Theatralized Critique of Market Aesthetics}

The performative dramaturgies of Olivier Choinière and

Guillaume Corbeil

\section{Crítica teatralizada de las estéticas mercantiles}
Las drramaturgias performativas de Olivier Choinière y Guillaume Corbeil

\section{Marie-Christine Lesage et Audrey-Anne Cyr}

Volume 39, numéro 1 (115), automne 2013

Théâtre et médias

URI : https://id.erudit.org/iderudit/1022991ar

DOI : https://doi.org/10.7202/1022991ar

Aller au sommaire du numéro

Éditeur(s)

Université du Québec à Montréal

ISSN

0318-9201 (imprimé)

1705-933X (numérique)

Découvrir la revue

Citer cet article

Lesage, M.-C. \& Cyr, A.-A. (2013). Critique théâtralisée des esthétiques marchandes : les dramaturgies performatives d'Olivier Choinière et de Guillaume Corbeil. Voix et Images, 39(1), 29-44.

https://doi.org/10.7202/1022991ar

\section{Résumé de l'article}

Cet article traite de Félicité et de Chante avec moi d'Olivier Choinière, deux oeuvres (l'une dramatique, l'autre scénique) au sein desquelles se cristallise l'entreprise artistique de ce créateur atypique, laquelle consiste, entre autres, à effectuer une critique des esthétiques marchandes. La perméabilité des rapports d'influence entre la réalité et le spectacle est au coeur de ces deux créations, dont la forme est inspirée par la pensée de Guy Debord.

L'importance accordée au rôle et à la position du spectateur est affirmée, chez Choinière, comme espace de désaliénation nécessaire. Cette traversée est complétée par une réflexion sur la pièce de Guillaume Corbeil, Nous voir nous (Cinq visages pour Camille Brunelle) (2013), qui prolonge autrement la critique d'un art de consommation de masse, en prenant comme modèle les modes de communication des réseaux sociaux. La toute récente dramaturgie de cet auteur s'attaque à l'hyperspectacle de soi dans la société médiatisée qui est la nôtre. Le texte se présente comme un dispositif performatif de paroles qui met en jeu les mécaniques aliénantes qu'il critique. Les créations de Choinière et de Corbeil ont la particularité d'inscrire à même leur architecture dramatique et scénique cette logique médiatique, en l'exacerbant de manière à en montrer le caractère aliénant, voire mortifère. 


\author{
C R I T I Q U E T HÉ ÂT R A L IS ÉE \\ DES ESTHÉTIQUES MARCHANDES \\ Les dramaturgies performatives \\ d'Olivier Choinière et de Guillaume Corbeil \\ $+++$ \\ MARIE-CHRISTINE LESAGE \\ Université du Québec à Montréal \\ AUDREY-ANNE CYR \\ Université du Québec à Montréal
}

Le théâtre québécois actuel semble loin d'avoir perdu sa force de frappe critique, si l'on en juge par quelques créations récentes qui interrogent, entre autres choses, les rapports individuels et collectifs que nous entretenons avec les industries culturelles de masse ainsi qu'avec l'hypermédiatisation de nos modes de communication. Nous nous intéresserons ici aux formes scéniques et dramatiques qui inventent moins des histoires sur le sujet qu'ils ne mettent en place des dispositifs performatifs. La notion de performativité, élaborée notamment par Richard Schechner ${ }^{1}$ et Philip Auslander ${ }^{2}$ aux États-Unis, a été reprise par Josette Féral pour désigner ce qu'elle nomme le théâtre performatif :

Cette notion met en valeur l'action elle-même plus que sa valeur mimétique de représentation. Elle remet en question le postulat qui veut que le théâtre soit indéfectiblement lié à l'imitation d'une action, à la représentation d'un sens, que celui-ci passe par les mots, par les gestes ou par l'image. Elle réfute l'idée que le thêâtre est nécessairement récit, narration, fiction et donc porteur de signification ${ }^{3}$.

À notre sens, un dispositif thêâtral performatif mettra donc en place une action concrète, un «faire scénique » impliquant nécessairement l'activité du spectateur; sur le plan dramatique, un texte-dispositif se présentera comme un agencement d'actes de parole et de situations sans que le tout soit nécessairement arrimé à une fable. Les créations scéniques hybrides d'Olivier Choinière (Chante avec moi et Mommy ${ }^{4}$ ) tout comme celles du collectif Petites cellules chaudes (Le iShow ${ }^{5}$ ) participent de cette forme du dispositif performatif; de même, les écritures d'un Étienne Lepage (Rouge

$$
++
$$

1 Richard Schechner, Performance. Expérimentation et théorie du thêâtre aux USA, traduit de l'anglais par Marie Pecorari et Marc Boucher, Paris, éditions Théâtrales, coll. «Sur le thêâtre», 2008, 532 p. 2 Philip Auslander, From Acting to Performance: Essays in Modernism and Postmodernism, Londres, Routledge, 1997, 173 p. 3 Josette Féral, Théorie et pratique du thêâtre. Au-delà des limites, Montpellier, Entretemps éditions, coll. "Champ thêâtral», 2011, p. 114. 4 Spectacles créés par Olivier Choinière, respectivement à l'Espace Libre (automne 2010) et au Thêâtre Aux Écuries (hiver 2013). 5 Spectacle créé par le collectif Petites cellules chaudes à l'Usine C (hiver 2013). 
gueule $^{6}$ ) ou d'un Guillaume Corbeil (Nous voir nous [Cinq visages pour Camille Brunelle $]^{7}$ ) déplacent de façon marquante les modalités de l'action dramatique.

Dans le cadre de cet article, nous nous pencherons sur des dispositifs théâtraux opérant une critique des diverses formes médiatiques qui concourent fortement à fabriquer les identités comme les imaginaires. Nous prendrons comme exemple Félicité ${ }^{8}$ et Chante avec moi d'Olivier Choinière, deux œuvres au sein desquelles se cristallise son entreprise artistique, laquelle consiste, entre autres, à effectuer une critique des esthétiques marchandes ${ }^{9}$. Nous terminerons cette brève traversée par une ouverture sur la pièce de Guillaume Corbeil, Nous voir nous (Cinq visages pour Camille Brunelle), laquelle prolonge autrement la critique d'un art de consommation de masse, en prenant comme modèle les modes de communication des réseaux sociaux. Les créations de ces deux auteurs ont la particularité d'inscrire à même leur architecture dramatique et scénique cette logique médiatique, l'exacerbant de manière à en démontrer le caractère aliénant, voire mortifère.

\section{FÉLICITÉ : LE JEU DES SIMULACRES}

Olivier Choinière est un inventeur de formes théâtrales qui cherche à déjouer tout ce qui asphyxie un art trop souvent contraint par des cadres institutionnels non seulement rigides, mais, plus essentiellement, inopérants sur le plan de la relation avec le spectateur. L'activité de ce dernier est au centre de la démarche du dramaturge, comme en témoigne d'ailleurs le nom de sa compagnie, qu'il a fondée en 2002 : l'Activité ${ }^{10}$. Celle-ci est décrite comme une "plateforme de création» qui interroge, remet en cause et redéfinit la représentation théâtrale et la place du spectateur dans la représentation. Selon Choinière, il importe que les préoccupations actuelles trouvent de nouveaux modes d'expression: " [La] réalité toujours nouvelle demande non seulement d'être portée par de nouveaux propos, mais aussi d'être incarnée par de nouvelles formes ${ }^{11}$.» Son parcours d'auteur et de concepteur artistique permet d'entrevoir le tracé d'une pensée exploratrice qui chemine suivant une continuelle réinvention formelle, laquelle est arrimée à un regard critique des plus décapants. À cet égard, il est intéressant d'observer que, depuis 1998, sa démarche s'est déplacée sensiblement de l'écriture dramatique (Jocelyne est en dépression, Venise-en-Québec, Félicité et Nom de domaine $^{12}$ ) vers une approche plus décloisonnée du théâtre, qui l'a mené, dès 2002,

$$
+++
$$

6 Étienne Lepage, Rouge gueule, Montréal, Dramaturges éditeurs, 2009, 107 p. 7 Guillaume Corbeil, Nous voir nous (Cinq visages pour Camille Brunelle), Montréal, Leméac, coll. "Théâtre/Leméac », 2013, 114 p. Désormais, les références à cet ouvrage seront indiquées par le sigle $N V N$ suivi du folio, et placées entre parenthèses dans le texte. 8 Olivier Choinière, Félicité, Montréal, Dramaturges éditeurs, 2007, 84 p. Désormais, les références à cet ouvrage seront indiquées par le sigle $F$ suivi du folio, et placées entre parenthèses dans le texte. $\mathbf{9}$ Le terme "esthétique marchande» est emprunté à Gilles Lipovetsky et Jean Serroy dans L'esthétisation du monde. Vivre à l'âge du capitalisme artiste (Paris, Gallimard, 2013, 493 p.). Désormais, les références à cet ouvrage seront indiquées par le sigle EM suivi du folio, et placées entre parenthèses dans le texte. 10 Sa compagnie est d'abord nommée: l'Activité Répétitive Grandement Grandement Libératrice (ARGGL!). 11 [S. a.], Olivier Choinière, en ligne: http://www.auxecuries.com/direction (page consultée le 27 juin 2013). 12 Olivier Choinière, Jocelyne est en 
à imaginer des projets de déambulations audioguidées (Beauté intérieure ${ }^{13}$, Bienvenue à [une ville dont vous êtes le touriste] ${ }^{14}$, Vers solitaire [OUT] ${ }^{15}$ ). Si ses œuvres dramatiques travaillent implicitement le regard du spectateur ( $c^{\prime}$ est particulièrement vrai de Félicité), les déambulatoires le mettent physiquement en jeu, afin de défamiliariser son rapport à l'espace urbain, tout en lui faisant entendre des partitions monologuées ${ }^{16}$. Dans ses créations récentes, Chante avec moi et Mommy, Choinière semble amorcer un nouveau volet exploratoire, qui pousse encore plus loin ce désir de trouver une forme qui soit à elle-même son propre contenu tout en ouvrant la représentation vers le spectateur, de façon à le projeter au centre de l'opération théâtrale.

La perméabilité des rapports d'influence entre la réalité et le spectacle est au fondement du travail d'Olivier Choinière. Il est intéressant d'observer à cet égard que Guy Debord s'impose dès Félicité comme une référence marquante. L'auteur met en exergue de sa pièce cette citation, tirée de La société du spectacle:

L'aliénation du spectateur au profit de l'objet contemplé (qui est le résultat de sa propre activité inconsciente) s'exprime ainsi: plus il contemple, moins il vit; plus il accepte de se reconnaître dans les images dominantes du besoin, moins il comprend sa propre existence et son propre désir. L'extériorité du spectacle par rapport à l'homme agissant apparaît en ce que ses propres gestes ne sont pas à lui, mais à un autre qui les lui représente. C'est pourquoi le spectateur ne se sent chez lui nulle part, car le spectacle est partout ${ }^{17}$.

Choinière inscrit de la sorte son texte dans la filiation de la pensée critique de Debord sur les médias et les esthétiques marchandes. L'importance accordée au rôle et à la position du spectateur au sein de ses créations thêâtrales est ainsi affirmée comme espace de désaliénation nécessaire. Avant même qu'il ne commence Félicité, le lecteur est mis en garde contre l'état de contemplation passive qu'induisent les images dominantes dans lesquelles il baigne quotidiennement. Pour Debord, le spectacle est la réalisation concrète de l'idéologie capitaliste, il vise la propagation d'une vision et de désirs uniformes, afin d'accroître la fonctionnalité économique de la société de consommation de masse. Son effet insidieux est de contaminer le réel au point de le dissoudre ${ }^{18}$, les simulacres l'emportant grâce à leur puissance de séduction et à leur capacité à fabriquer du rêve en boîte tout en stimulant un certain voyeurisme de compensation. Et c'est précisément cet espace de disparition du réel que travaille la pièce Félicité, un réel qui apparaît phagocyté par les simulacres créés par le star system

$$
+++
$$

dépression. Tragédie météorologique, Montréal, Dramaturges éditeurs, 2002, 67 p. ; Venise-en-Québec. Épopée touristique, Montréal, Dramaturges éditeurs, 2006, 168 p.; Nom de domaine, Montréal, Leméac, coll. "Théâtre/ Leméac», 2012, 70 p. 13 Olivier Choinière, Beauté intérieure, Montréal, Dramaturges éditeurs, 2003, 99 p. 14 Créé par Olivier Choinière (ARGGL!), Montréal, 2005. 15 Créé par Olivier Choinière (L'Activité), Théâtre La Chapelle, 2008. 16 Sur les déambulatoires, voir Francis Ducharme, "Quand le théâtre joue à se prendre pour du tourisme: les déambulatoires audioguidés d'Olivier Choinière ", L'Annuaire théâtral, no 47, printemps 2010, p. 85-101, et "Seul à seul avec une voix dans la ville. Beauté intérieure et Vers solitaire (OUT) 》, Jeu. Revue de thêâtre, n 147, 2013, p. 88-94. 17 Guy Debord, La société du spectacle, Paris, Buchet/Chastel, 1967, p. 20. 18 C'est aussi la thèse, bien connue, de Jean Baudrillard, développée notamment dans son livre Simulacres et simulation (Paris, Galilée, 1981, 233 p.). 
américain et les médias populaires. Au centre de la pièce règne, en effet, la figure emblématique de Céline Dion, pur produit d'une esthétique marchande qui croise le spectaculaire et l'impératif commercial. Céline Dion est à elle-même une industrie, une marque de parfum, un label musical; elle est aussi une figure qui fait partie de notre "patrimoine culturel», tant chacun connaît l'histoire de son enfance, de sa famille et de sa fulgurante ascension (planifiée par un homme d'affaires) vers un succès mondialisé. Choinière s'adresse directement à la communauté de spectateurs d'ici, sa critique du star system américain et du journalisme à sensation s'incarnant dans des figures connues et familières du public. Mais la force performative de la pièce, c'est-à-dire son action expérientielle sur le lecteur comme sur le spectateur, réside dans sa composition dramatique, laquelle met en œuvre un trajet perceptif qui fait vivre ce dont il est question; autrement dit, le spectateur est amené à suivre de l'intérieur l'effet de déréalisation engendré par l'hypermédiatisation du monde.

Félicité orchestre trois récits qui se suivent, s'entremêlent et se contaminent progressivement, suivant un principe de digression: le passage d'un récit vers un autre s'opère par une focalisation sur un détail, qui ouvre sur un autre récit, lequel reprend un motif du précédent, mais en le transposant dans un tout autre contexte, avec d'autres personnages. La pièce débute avec la narration des faits entourant le dernier spectacle de Céline Dion, après qu'elle a annoncé qu'elle attendait un enfant. Quatre personnages prennent en charge cette histoire, qui est entièrement rapportée de façon chorale ${ }^{19}$, selon un mode qui allie le narratif et le dramatique, les quatre voix alternant pour décrire le même événement, celui des adieux de Céline Dion. La description des personnages indique qu'il s'agit d'employés d'un magasin à grande surface (un Walmart, apprendra-t-on à la toute fin de la pièce) : on retrouve la préposée, l'étalagiste, le gérant, auxquels se joint l'oracle, qui se révèle être celle qui amorce et dirige en partie les récits:

ÉTALAGISTE: CÉLINE à la télévision - dans toutes les maisons

PRÉPOSÉE: Dans le salon - le monde, ça pleurait

ÉTALAGISTE: Le lendemain - les journaux, les magazines: «ADIEU, CÉLINE», écrit en grosses lettres

ORACLE: Pas «ADIEU», «Ā BIENTÔT »

GÉRANT : «Ả BIENTÔT » - c'est ça oui - Céline voulait juste prendre une pause. PRÉPOSÉE: Quand Céline est apparue pour la conférence de presse, en jeans pis en chandail - un chandail ample, tellement - simple. Les journalistes en sont tombés à genoux. $(F, 13)$

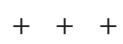

19 La notion de choralité est employée au sens récent du terme: «a minima, on entend par choralité cette disposition particulière des voix qui ne relève ni du dialogue, ni du monologue; qui, requérant une pluralité (un minimum de deux voix), contourne les principes du dialogisme, notamment réciprocité et fluidité des enchaînements, au profit d'une rhétorique de la dispersion (atomisation, parataxe, éclatement) ou du tressage entre différentes paroles qui se répondent musicalement (étoilement, superposition, échos, tous effets de polyphonie)». Martin Mégevand, "Choralité», Jean-Pierre Ryngaert (dir.), Nouveaux territoires du dialogue, Arles/Paris, Actes Sud/Conservatoire national supérieur d'art dramatique, coll. «Apprendre», 2005, p. 37-38. 
Choinière situe sa pièce dans le monde de la vente d'objets de consommation de masse, afin de traiter de l'aliénation qu'entraîne la production de l'industrie des arts de consommation populaire qui lui est liée. Toutefois, il recourt à une stratégie dramatique très habile: en ouverture, les quatre personnages entrent et se placent face au public avec, sur leur tablier bleu, une épinglette à leur nom, mais dont les lettres sont à l'envers. De la sorte, il est signifié visuellement au spectateur que la scène se déroule "de l'autre côté du miroir», comme cela est par ailleurs indiqué dans l'incipit. En effet, la pièce nous introduit dans l'univers fantasmatique imaginé par le personnage de l'oracle, celle qui a des visions, en réalité une caissière répondant au nom de Caro et dont le numéro d'employé est 31 . Les récits portés par les quatre personnages sont en vérité des inventions de Caro/Oracle, qui intervient d'ailleurs ponctuellement afin de rectifier la version des faits racontés ou de rediriger le récit. De surcroît, les paroles rapportées sont en partie empruntées à des médias comme les journaux à potins et les conférences de presse de vedettes, Choinière indiquant ces emprunts par l'utilisation de majuscules dans le texte:

ÉTALAGISTE: Vêtement traditionnel, mais je dis pyjama parce que c'était si - simple, si beau de les voir, de voir tout à coup l'homme et la femme, comme n'importe quel couple qui se lève, "CÉLINE ET RENÉ FONT LA GRASSE MATINÉE», disait la légende. Des futurs parents qui s'aiment et qui attendent un bébé. $(F, 20)$

Ces emprunts témoignent du degré d'infiltration du star system médiatique dans l'imaginaire de Caro, cette dernière étant ainsi transformée en oracle de Céline : la jeune caissière transmue le réel qui est le sien en une suite de visions fantasmatiques qui la dépossèdent de toute forme d'individuation (les médias de masse passent et parlent à travers elle), tout en l'aliénant de son rapport au réel (ses collègues de travail deviennent les figures parlantes imaginaires de ce monde de simulacres intérieurs). Caro est imprégnée du discours des médias de masse et sa relation intérieure à l'icône médiatique au centre de son univers, Céline Dion, correspond à ce qu'expliquent Gilles Lipovetsky et Jean Serroy à propos des vedettes de cinéma et de la chanson populaire : la star « apparaît comme une réalité entre figure publique et figure privée» $(E M, 212)$; elle est créée par le marketing de masse pour faire rêver le plus grand nombre, mais l'intérêt qu'on lui témoigne est aussi « de type extra-esthétique, parce qu'il porte sur la vie personnelle et intime. Le fan s'intéresse sans doute moins aux films où joue la star qu'il adule qu'à tout ce qui est en dehors de ceux-ci (ses goûts personnels, sa vie familiale, ses liaisons,...)» $(E M, 212)$. C'est précisément cette fascination pour la vie privée de Céline Dion qui est représentée ici, Caro ingérant littéralement la vie hypermédiatisée de cette dernière.

La pièce thématise donc la question des esthétiques marchandes dont la visée est de produire de la fascination, de la beauté, du plaisir, afin d'alimenter le désir de consommation. Mais elle en fait vivre les dérives par sa composition formelle, qui orchestre un jeu de torsions narratives incarnant l'aliénation intérieure du personnage de Caro. Au fil du récit portant sur la grossesse de Céline, l'oracle introduit de nouveaux faits, qui vont progressivement faire dévier l'histoire de la chanteuse populaire vers une tout autre situation, beaucoup plus sordide, laquelle semble directement 
issue des journaux à sensation. L'embranchement du récit initial vers le second se produit après que Céline a perdu son bébé dans un flot de sang: l'oracle indique aux autres personnages, à trois reprises, "Ça sonne à la porte», puis «C'est la voisine». Sa parole est l'embrayeur de la bifurcation diégétique qui s'amorce alors, les autres personnages interrogeant l'oracle afin de connaître le nouveau sens de l'histoire:

ORACLE: Ça sonne à la porte. C'est la voisine.

PRÉPOSÉE: Quelle voisine?

ORACLE: La voisine d'à côté.

PRÉPOSÉE: La Mère de Céline savait pas que Céline et René avaient des voisins.

$(F, 33)$

Cet échange crée l'impression d'un récit se fabriquant au fur et à mesure sous la conduite de l'oracle, ce qui accentue l'impression d'entrer dans un monde de simulacres intérieurs. À partir de ce moment, le récit de Céline se transforme graduellement en celui d'une jeune fille nommée Isabelle, la Mère de Céline devenant la Mère d'Isabelle, le Père de Céline, le Père d'Isabelle, et ainsi de suite. Le glissement d'un récit à l'autre se révélera être le croisement de deux nouvelles lues, tout autant que vues, dans un journal à sensation qui parle de Céline Dion et de Star Académie sur une page, et qui sur l'autre montre la photo d'un père iraquien avec son fils mort dans les bras, puis une nouvelle locale sordide, relatant la mort d'une jeune fille qui a été négligée et abusée par ses parents et son frère. Le personnage d'Isabelle semble être le fruit d'un amalgame, créé par l'imaginaire de Caro/Oracle, entre la jeune fille de cette nouvelle et Caro elle-même, car elle fait d'Isabelle la plus grande admiratrice de Céline Dion:

ORACLE: Ferme les yeux. Maintenant, imagine la vie que t'aurais si t'étais toujours couchée. Imagine la vie d'Isabelle, la fan de Céline, couchée dans sa chambre, sa chambre de fille malade, les rideaux toujours fermés - à des années-lumière de la chambre de son idole à Las Vegas. $(F, 45)$

Le récit des personnages plonge alors le lecteur/spectateur dans l'univers d'une misère extrême, lui détaillant les sévices subis par Isabelle, laquelle est maltraitée, malade, abusée sexuellement, ne survivant, enfermée dans sa chambre, que grâce à Céline. Mais cette Isabelle fantasmée à partir d'une nouvelle à sensation, nous l'avons dit, $c^{\prime}$ est aussi la figure de Caro qui, tout comme Isabelle, ne serait rien sans Céline, sinon «juste un corps sans nom» $(F, 46)$. Or, il s'avère que Caro est une employée qui prend ses pauses seule dans les toilettes et que tous les autres employés fuient. C'est ce que révèle le troisième et dernier récit, qui constitue en fait un retour à la réalité du Walmart où travaillent Caro et les autres voix-narratrices: la mort d'Isabelle, qui se vomit tout le corps et les organes, devient l'élément déclencheur qui fait basculer le récit la concernant vers celui du quotidien des personnages-narrateurs, lesquels, pour la première fois, vont endosser leur véritable rôle.

Pour ce qui est du partage des voix, ce troisième récit se présente différemment, le gérant, la préposée et l'étalagiste rapportant cette fois leurs propres pensées, actions 
et échanges avec Caro. Ainsi glisse-t-on d'Isabelle qui se vide l'intérieur vers Caro en train de vomir son lunch dans les toilettes du Walmart, sous le regard de la préposée. Le corps qui se vomit les entrailles est une image organique forte rappelant ces êtres vidés de leur substance par le spectacle médiatisé du monde, lequel s'est infiltré dans leur intimité. Caro/Oracle ne porte en elle que des récits médiatiques de vedettes et de faits divers à partir desquels elle bricole et imagine des histoires qui s'enchevêtrent et auxquelles elle entremêle les figures de sa réalité. Un miroir trouble et troublant est créé entre l'histoire de Céline Dion, celle d'Isabelle et l'existence de Caro, ce jeu des simulacres faisant en sorte que le réel du personnage est noyé dans une galerie d'images sans fond. De plus, l'histoire sordide d'Isabelle apparaît comme l'excroissance monstrueuse, hyperbolique, de la violence engendrée par la médiatisation d'une culture populaire de masse, qui digère toute possibilité d'individuation en réduisant chacun à l'état de contemplateur passif et aliéné des images de ce monde du spectacle. En ce sens, Caro en Oracle incarne, pour Choinière, la conséquence de l'aliénation selon Debord : elle ne s'appartient plus, elle est intérieurement cette Isabelle enchaînée, impotente et vidée d'elle-même, abusée par un système qui la dévore. La démonstration de l'auteur ${ }^{20}$ emprunte la figure grotesque du monstrueux et de l'organique, un peu comme a pu le faire Sarah Kane dans L'amour de Phèdre ${ }^{21}$, avec un Hippolyte obèse, avachi et amorphe devant son téléviseur. Il suggère ainsi qu'Isabelle est l'enfant avorté de Céline Dion, ce dernier apparaissant comme la conséquence inéluctable d'une esthétique marchande abrutissante. Ainsi s'incarne thêâtralement l'idée de Debord selon laquelle «l'extériorité du spectacle par rapport à l'homme agissant apparaît en ce que ses propres gestes ne sont pas à lui, mais à un autre qui les lui représente ${ }^{22} »$.

Le partage des voix et l'imbrication des récits permettent au lecteur/spectateur de ressentir cette impression de perte du réel ou, à tout le moins, de confusion entre le réel et ses simulacres. Il est intéressant de noter que la forme de Félicité, où les récits paraissent s'inventer au fur et à mesure, n'est pas sans rappeler les pièces de l'auteur britannique Martin Crimp qui, dans Atteintes à sa vie ${ }^{23}$, Face au mur, ou encore Tout va mieux ${ }^{24}$, recourt à ce type de dialogue aux énonciateurs incertains, lesquels travaillent ensemble à inventer un récit. Si Choinière met Debord en exergue de sa pièce, Crimp choisit Baudrillard pour Atteintes à sa vie: "Qu'en est-il alors de l'événement réel si partout l'image, la fiction, le virtuel perfusent dans la réalitée ${ }^{25}$ ?»

20 Démonstration, car la forme dramatique met en place une certaine rhétorique ou une "praxis argumentative», au sens où l'entend Gilles Declercq à propos du drame contemporain : c'est dans la macrostructure de l'ensemble de la pièce qu'opère la force argumentative, laquelle se caractérise par « une rhétorique extrascénique fondée sur le travail herméneutique du spectateur» («Rhétorique et dialectique du dialogue», Jean-Pierre Ryngaert [dir.], Nouveaux territoires du dialogue, p. 60). La pièce est un dispositif qui démontre, en la mettant en œuvre, la manipulation des personnes par le système médiatique; le lecteur/spectateur a cependant à sa charge d'interpréter cette macrostructure dramatique. On peut dire que la référence initiale à Debord annonce ce qui sera démontré dans Félicité. 21 Sarah Kane, Manque. L'amour de Phèdre, Paris, L'Arche, coll. "Scène ouverte», 1999, 119 p. 22 Guy Debord, La société du spectacle, p. 20. 23 Martin Crimp, Atteintes à sa vie, traduit de l'anglais par Christophe Pellet, Paris, L’Arche, coll. «Scène ouverte», 2009 [1997], 96 p. 24 Martin Crimp, Face au mur. Tout va mieux, Paris, L'Arche, coll. «Scène ouverte», 2004, 53 p. 25 Crimp cite Jean Baudrillard (Simulacres et simulations, 1981) en exergue de la pièce (Martin Crimp, Atteintes à sa vie, p. 9). 
Selon le philosophe, l'image consomme l'événement; aussi est-ce précisément ce que travaillent les pièces récentes de Crimp, qui font entendre les voix de ce qui s'apparente à des créateurs publicitaires fabriquant les histoires et images médiatisées déterminant ensuite notre perception du monde. Ces textes mettent en œuvre des dispositifs dialogiques inédits, qui fonctionnent suivant trois modes principaux ${ }^{26}$ : l'autoengendrement du récit, les digressions et une logique expansive. Les personnages de ces pièces sont des énonciateurs anonymes qui font fluctuer un récit, lequel donne l'impression de se tisser au fur et à mesure qu'on le raconte. On observe un procédé de décentrement constant du récit principal vers une description, un commentaire ou un autre récit; les histoires mènent toujours à des événements violents dont on ne sait s'ils sont réels ou imaginaires. Enfin, on a l'impression que c'est la multiplication des potentialités d'une même situation dramatique qui prime, la logique de digression faisant perdre son unicité au récit dramatique. On retrouve toutes ces composantes dans la forme dramatique de la pièce de Choinière. Aussi n'est-il pas étonnant que Félicité ait été traduite par Caryl Churchill et jouée au Royal Court Theatre de Londres, le théâtre qui a accueilli les premières créations des œuvres de Kane et de Crimp : la pièce de Choinière s'inscrit clairement dans cette filiation d'une dramaturgie britannique qui critique l'hypermédiatisation du monde, mais en inventant une forme dramatique qui témoigne aussi de celle-ci.

\section{CHANTE AVEC MOI : SPECTACULARISATION ET DISPOSITIF PERFORMATIF}

Chante avec moi s'inscrit dans la continuité de la critique ouverte par Félicité, mais Choinière choisit cette fois d'inventer un dispositif ${ }^{27}$ performatif qui met en œuvre le système spectaculaire qu'il critique, afin d'en épuiser la mécanique. Cette création est fondée sur la reprise en continu de la même chanson, dont l'air accrocheur agit comme un véritable ver d'oreille, tant il s'infiltre dans l'esprit à la manière des tubes composés dans l'industrie de la musique commerciale. Le spectacle, essentiellement musical, est composé de quatre parties, qui reprennent le même tube avec des variations de style. Le début se présente comme une fausse improvisation musicale, qui donne aux spectateurs l'impression qu'ils assistent en direct à la fabrication de la nouvelle chanson à succès. Tour à tour, des performeurs qui étaient assis dans la salle se lèvent et se rendent sur la scène pour contribuer à la création de la chanson en direct, ce qui fait en sorte que, dès le départ, elle se présente comme un bien commun appartenant à tous

$$
++
$$

26 Voir Rachel Spengler, «Une dramaturgie du décentrement. Étude de Atteintes à sa vie, Tout va mieux et Face au mur de Martin Crimp ", L'Annuaire théâtral, n 38, automne 2005, p. 53-67. 27 La notion de dispositif apparaît plus adéquate que celle de représentation pour désigner la forme spectaculaire inventée par Choinière. En effet, le dispositif met l'accent sur l'expérience du spectateur, il lui propose un agencement événementiel plus qu'un univers fictionnel, et il implique une certaine contrainte qui peut être d'ordre technique ou autre. $\bar{A}$ ce sujet, voir Giorgio Agamben, Qu'est-ce qu'un dispositif?, traduit de l'italien par Martin Rueff, Paris, Payot \& Rivages, coll. «Rivages poche/Petite bibliothèque», 2007 [2006], 50 p. ; Arnaud Rykner, « Du dispositif et de son usage au thêâtre», Tangence, nº 88, automne 2008, p. 91-103. 
les spectateurs. Le refrain de la chanson, accrocheur et facilement reconnaissable, interpelle d'emblée le public: «Je chante. Oui je chante. Pour que tu chantes avec moi. » Il utilise tous les ingrédients de la chanson populaire: des phrases simples, la répétition de mots aisément mémorisables et une musique enjouée, rythmée, avec des crescendo d'intensité. La reprise de la chanson (qui devient ritournelle) contribue à l'inclusion progressive du public dans la machine spectaculaire. Le tour de force de cette création réside justement dans cet art de prendre le spectateur au piège de son désir de divertissement ou, à tout le moins, de lui faire vivre l'expérience de la mécanique aliénante de l'industrie du spectacle.

Si la première partie adopte la forme caractéristique de la comédie musicale américaine (sur le modèle des musicals de Broadway), la variation introduite en seconde partie évoque davantage l'ère des spectacles à grand déploiement, avec costumes design, éclairages frappants et décors extravagants, et dont le but principal est d'éblouir les spectateurs, de leur en mettre plein la vue: on pense à Pink Floyd, au Cirque du Soleil, à Madonna, à Céline Dion, pour ne nommer que quelques figures emblématiques de l'industrie du mégaspectacle. À la fin de cette seconde partie, qui cherche l'adhésion totale du public (en faisant tomber ses dernières défenses critiques), un nouveau punch est introduit: chaque soir, une vedette de la chanson populaire se joint aux performeurs, afin de chanter avec eux sur scène ${ }^{28}$. Cette incursion d'une figure réelle de l'industrie du spectacle a comme conséquence de provoquer immédiatement un effet de surprise dans le public, qui accueille alors la vedette avec une émotion bien sentie. À ce moment, la spectacularisation du dispositif atteint son zénith, en ce que la majorité des spectateurs est conquise par l'extraordinaire énergie du show et par la légèreté rassembleuse du tube. Choinière réussit à recréer, à plus petite échelle, l'impact des spectacles à grand déploiement qui produisent sur le public cette impression de participer à un moment magique; le divertissement vise le consensus, la communion, la participation collective à un événement qui se présente comme étant unique (alors qu'il relève toujours d'une machine spectaculaire planifiée dans ses moindres détails) et qui ne vise qu'une chose: stimuler le plaisir, les émotions, la fascination du plus grand nombre. Les esthétiques marchandes, si elles sont fondées sur la participation collective, n'offrent en contrepartie aucune valeur symbolique de partage, sinon celle de la consommation de l'événement pour sa seule valeur distractive. La dramaturgie performative à laquelle recourt Choinière emprunte aux codes des spectacles musicaux populaires, en travaillant initialement la complicité avec le spectateur (nous participons ensemble à la création en direct de la chanson, qui nous rassemble), puis en accroissant la courbe de son plaisir par le spectaculaire de la forme et l'arrivée d'une vedette surprise. Le couperet critique tombe toutefois à la troisième répétition-variation de la chanson: d'un spectaculaire à outrance, on passe à une condamnation lucide de l'industrie du divertissement musical. La puissance performative du dispositif imaginé par Choinière commence alors son opération et elle est d'autant plus effective que le spectateur aura adhéré (à force de répétitions) aux premières variations de la chanson mise en scène. Dans cette partie, les performeurs,

Daniel Bélanger était l'invité le soir où nous avons assisté à l'événement. 
épuisés, reprennent l'enchaînement de paroles et de mouvements, suivant un rythme de plus en plus effréné; leur gestuelle se fait mécanique, ils se transforment en de sombres pantins obéissants, qui chantent jusqu'à l'épuisement et la mort (des techniciens de scène sortent alors un à un ceux qui s'écroulent). Ils sont contraints de poursuivre leur manège aussi longtemps que l'assistance les applaudira: alors qu'ils ont terminé leur séquence, la salle applaudit la «fin» du spectacle, mais chaque battement de mains redémarre la machine spectaculaire et force les performeurs (ceux qui tiennent encore) à reprendre leur danse, même s'ils n'en peuvent plus. Ce manège vise la prise de conscience, par le spectateur, de son rôle dans la mécanique de cette esthétique marchande qu'il consomme et qui évide autant l'art que les individus qu'elle enchaîne. Aussi faut-il qu'il cesse enfin d'applaudir pour que s'arrête la ritournelle! Non seulement le public fait-il alors partie du rouage spectaculaire, mais il est ainsi mis devant une double contrainte: s'il n'applaudit pas la fin du spectacle, il déroge aux codes de la représentation qui demandent que l'on salue de la sorte les artistes pour leur performance dans Chante avec moi; s'il le fait, il prend le rôle du consommateur qui participe volontairement à une industrie mortifère et aliénante. Autrement dit, Choinière crée un dispositif qui déborde la représentation, en éliminant le cadre extrascénique de Chante avec moi : le public est pris au piège d'un dispositif dont il ne peut sortir qu'en cessant volontairement d'y participer. Aussi était-il intéressant d'observer dans la salle la force de la tentation, pour une majorité, de continuer à battre des mains à l'unisson, bien que l'image scénique des danseurs épuisés fît de plus en plus penser à des soldats embrigadés. La métaphore d'un système totalitaire et aliénant pour les individus, les enchaînant à la machine industrielle d'un travail à la chaîne forcé (rappelant en cela le fordisme), au service d'une société du spectacle commerciale, se faisait pourtant clairement jour.

Enfin, Choinière en rajoute avec un appendice, dernière partie de son dispositif performatif: alors que tous croient que Chante avec moi est terminé, il revient à la charge en installant un iPhone à l'avant-scène, sur lequel va être rejouée la version enregistrée du spectacle. La nouvelle scène de la société du spectacle ainsi désignée est celle des nouveaux médias, grâce auxquels tous les événements vécus sont enregistrés et diffusés au plus grand nombre d'internautes: l'iPhone est devenu le média qui témoigne de notre rapport à l'événementiel, au point de se substituer à l'expérience réelle (je filme l'événement au lieu de le vivre, puis je regarde et partage en réseau l'image de ce qui a eu lieu devant moi... presque sans moi). Choinière met à mort le spectaculaire de l'industrie du spectacle pour mieux présenter, à la fin, sa version contemporaine: l'iPhone comme objet symbolique de la culture médiatique actuelle. YouTube, les blogues, Facebook participent aujourd'hui à cette industrie de la musique et du spectacle, qui a adapté ses modes de diffusion à ce monde médiatique. Le caractère portatif de l'iPhone remet radicalement en question les critères de consommation et de réception du spectacle. Il redéfinit l'essence même de ce dernier, qui ne se construit désormais plus nécessairement par la rencontre entre performeurs et spectateurs: on peut aujourd'hui se faire une culture spectaculaire uniquement à partir de YouTube...

Chante avec moi présente, en somme, des variations scéniques qui témoignent de l'évolution historique des formes spectaculaires des esthétiques marchandes, les- 
quelles pactisent aujourd'hui avec les nouvelles technologies. En retournant comme un gant, dans les deux dernières parties de Chante avec moi, la logique du spectaculaire, Choinière renoue avec les propos de Guy Debord sur l'aliénation du public rendu passif par la contemplation de divertissements faciles. Cela dit, lors de la représentation à laquelle nous avons assisté, un curieux phénomène collectif s'est produit: le public, devant le faible volume sonore de l'iPhone, s'est mis à chanter le refrain de la chanson en chœur, à notre grand étonnement. Signe que la performativité critique du dispositif n'avait pas opéré de la même manière pour tous?

\section{NOUS VOIR NOUS (CINQ VISAGES POUR CAMILLE BRUNELLE): L'HYPERSPECTACLE DE SOI}

L'iPhone sur la scène de Chante avec moi dénonçait clairement l'empire de la culture médiatique actuelle ${ }^{29}$ comme nouvelle scène de la société du spectacle. Tant dans Félicité que dans Chante avec moi, c'est en effet la société du spectacle telle que décrite par Debord que cible Choinière. Aujourd'hui, à l'ère du numérique, la culture subit des transformations majeures quant aux modèles de production, de diffusion et de consommation. L'omniprésence des médias numériques transforme les modes de réception culturels : Internet et tous les réseaux sociaux prennent une telle place dans la diffusion qu'il devient pratiquement impossible de distinguer la culture des médias. Les sociologues Éric Macé et Éric Maigret ont avancé un nouveau terme pour désigner ce phénomène: les «médiacultures ${ }^{30}$ ». L'émergence de nouveaux supports dématérialise en fichiers numériques le contenu de la culture, qui devient ainsi aisément accessible virtuellement. La thèse du dernier livre de Gilles Lipovetsky et Jean Serroy, L'esthétisation du monde, tend également à démontrer que la société du spectacle telle que pensée par Debord a franchi un cap inédit sous l'empire des nouveaux médias. Selon les auteurs, il n'est plus de domaine qui échappe aux logiques poussées à l'extrême de l'image et du divertissement:

le capitalisme artiste a créé un empire transesthétique proliférant où se mélangent design et star system, création et entertainment, culture et show business, art et communication, avant-garde et mode. Une hyperculture communicationnelle et commerciale qui voit s'éroder les classiques oppositions de la fameuse «société du spectacle» $(E M, 27)$.

Cette hypermédiatisation de la culture (haute et populaire confondues sur les mêmes plates-formes) remettrait en cause la problématique classique développée par l'école de Francfort et Debord, selon laquelle la puissance du médiatique engendre passivité, aliénation et dépossession de soi chez les individus:

29 Dans sa dernière pièce, Nom de domaine, Olivier Choinière s'attaque à l'univers virtuel, en mettant en scène une famille qui joue, sans le savoir, au même jeu interactif violent. 30 Éric Maigret et Éric Macé (dir.), Penser les médiacultures. Nouvelles pratiques et nouvelles approches de la représentation du monde, Paris, Armand Colin, coll. «Médiacultures», 2005, 186 p. 
Au vrai, à l'ère du capitalisme artiste hypermoderne, il y a bel et bien instrumentalisation par les individus du monde écranique spectaculaire. On est dans l'hyperspectacle lorsque, au lieu de «subir » passivement les programmes médiatiques, les individus fabriquent et diffusent en masse des images, pensent en fonction de l'image, s'expriment et portent un regard réflexif sur le monde des images, agissent et se montrent en fonction de l'image qu'ils veulent voir projetée d'eux. (EM, 275)

Autrement dit, les individus ne font pas que subir le système médiatique, ils s'en servent afin de se mettre eux-mêmes en scène. C'est précisément le sujet de la pièce de Guillaume Corbeil, Nous voir nous (Cinq visages pour Camille Brunelle), dans laquelle il interroge cette ère de l'hyperspectacle du moi, qui est à distinguer de la critique debordienne de la société du spectacle.

La pièce de Corbeil fait entendre cinq voix (Un, Deux, Trois, Quatre et Cinq) qui s'agencent (plus qu'elles ne dialoguent) en empruntant les codes de communication propres aux réseaux sociaux. D'entrée de jeu, les personnages se décrivent à tour de rôle (âge, sexe, couleur des yeux et des cheveux, taille, date de naissance, statut: tous célibataires) et présentent leurs goûts en matière de vêtements, de passe-temps, de musique, de films, de livres et de théâtre, suivant le modèle du «I like». Les répliques sont composées de listes s'apparentant à celles qui sont publiées sur une page Facebook, et il y a une logique de surenchère et de rivalité entre les voix:

UN : J'aime aller voir les groupes que j'aime en concert

TROIS : J'aime découvrir des nouveaux groupes

UN : J'aime écouter un album que je connais pas d'un bout à l'autre

TROIS : J'aime aller voir des groupes que je connais pas en concert

Acheter leur disque

Et l'écouter d'un bout à l'autre

QUATRE: J'aime The Buzzcocks

J'aime Pavement

J'aime The Smiths

J'aime Jon Spencer Blues Explosion

J'aime The Dead Weather

J'aime We are Wolves

J'aime The Breeders

TROIS : J'aime The Pixies

J'aime Animal Collective

J'aime Of Montreal

J'aime Pulp

J'aime Department of Eagles

UN : J'aime The Black Eyed Peas

J'aime La Roux

J'aime DJ Shadow 
J'aime Cypress Hill

J'aime M.I.A.

J'aime Aphex Twin

J'aime Yelle

J'aime Michael Jackson

J'aime Madonna. (NVN, 19-20)

Le premier tableau de la pièce (qui en compte cinq) fonctionne sur ce modèle du listage des goûts personnels, alignant les références culturelles et artistiques selon un mode totalisant. En effet, l'étalage des œuvres vues, écoutées et lues est quantitatif, rien n'étant transmis quant à la relation sensible que chacun entretient avec celles-ci. Aussi les personnages apparaissent-ils comme des hyperconsommateurs culturels qui auraient tout absorbé de l'art moderne et contemporain, et ce, sans distinction de styles ni de genres, les classiques croisant les formes contemporaines, les formes populaires se mêlant à l'art plus élitaire suivant cette idée d'une médiaculture qui entremêle tout. Le capitalisme artiste transesthétique décrit par Lipovetsky et Serroy est parfaitement illustré par ces listes - énoncées dans le but de projeter une image branchée de soi -, qui associent indistinctement Nelly Arcan, Catherine Millet, Antoine de Saint-Exupéry et Albert Camus. Cette génération connectée à Google, iTunes et Wikipédia a accès à tout un stock culturel aisément consultable sur les sites Internet, qu'elle consomme en masse sans toutefois effectuer de discrimination quant à la valeur et au sens des œuvres, dont elle ne retient par ailleurs que les titres. La logique de surenchère qui anime les dialogues tout au long du premier tableau montre de façon éloquente que ces individus ont adopté, comme mode d'interaction sociale, celui qui caractérise l'hyperspectacle:

c'est une logique hypertrophique de surenchère, de «toujours plus» qui constitue l'hyperspectacle. Dans ce système où les signes ne renvoient qu'à eux-mêmes sans autre finalité que l'impact spectaculaire, médiatique et marchand, nous sommes témoins d'une orgie d'artifices, de paillettes et d'effets publicitaires, d'événements surmédiatisés et émotionnels, d'extravagances et d'images extrêmes (EM, 276).

Les cinq figures de Nous voir nous participent à cette logique, qui est aussi celle des réseaux sociaux, lesquels constituent les modes actuels d'interaction entre les êtres, même les plus intimes. L'usage de ces réseaux en ligne modèle les formes de la socialisation, les échanges interindividuels adoptant les stratégies imposées par l'hypermédiatisation. La suite de la pièce illustre les conséquences ultimes de ce système, qui mène à l'hyperspectacularisation du moi, chacun mettant en ligne et en image tout ce qu'il vit, de manière à fabriquer et à diffuser une certaine marque de commerce de soi.

Au second tableau, on retrouve les cinq personnages après une soirée mémorable, dont ils se remettent en se montrant (ou en s'envoyant par Facebook) les photos de la veille, qui témoignent de leur virée «super » et "malade». Aucune indication de lieu n'étant donnée, les dialogues peuvent se produire dans un espace réel ou sur un site en réseau. Les événements sont tous rapportés à partir des images prises sur un iPhone, suppose-t-on: 


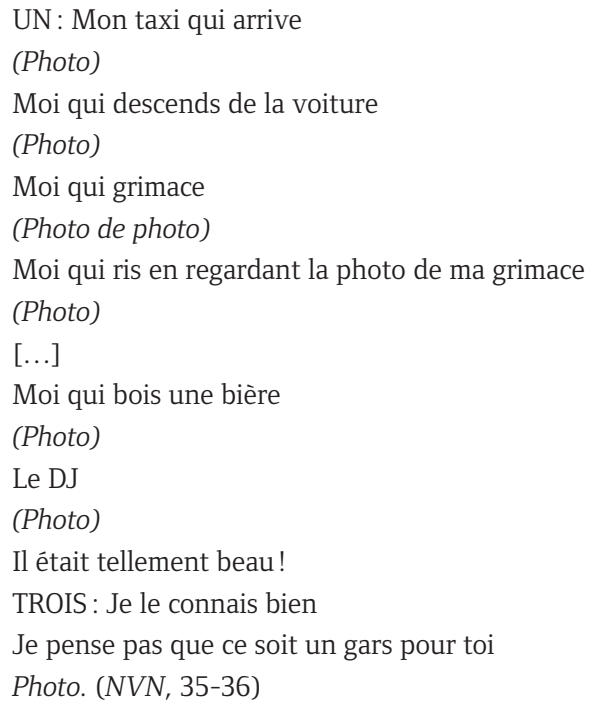

Toutes les situations rapportées par les personnages, et qui témoignent de leur passé immédiat, le sont par des photos, des photos de photos, des photos d'images, et ainsi de suite. On remarque que certaines indications sont entre parenthèses et d'autres pas, ce qui distingue les images montrées de celles prises dans le présent du locuteur (la mise en images ne cessant ainsi jamais). Les interactions (réelles ou virtuelles) entre les cinq amis sont médiatisées par les photos prises la veille, celles-ci témoignant d'un événement vécu dans la conscience d'une autoreprésentation de soi, d'une mise en scène du moi pensée en fonction des images, qui vise à manifester en permanence son existence sur le mode d'un star system où la vedette est à elle-même son propre paparazzi : «la société du spectacle était centrée sur les stars mythiques du cinéma et de la chanson; celle de l'hyperspectacle est contemporaine d'une espèce de starisation généralisée s'appliquant à toutes les activités» (EM, 277). Avec les nouveaux médias apparaît l'hyperspectacle du moi qui rejoue sur le plan intime l'économie du vedettariat et des marchés de la renommée; d'où la pertinence de la citation d'Elfriede Jelinek, mise en exergue de la pièce: «Dans toutes ces averses de flashs, sans aucune intimité, je suis tout à fait intime, en étant tout à fait publique, l'un n'enlève rien à l'autre ${ }^{31}$. " $(N V N, 9)$ Ce désir des personnages d'exhiber leur vie intime, de la «stariser», se fait aussi sur le mode de la surenchère expérientielle caractéristique du spectaculaire médiatique. Chacun rivalise en effet, grâce à une accumulation exponentielle de photos, d'images et de vidéos, d'aventures plus extraordinaires et sensationnelles les unes que les autres: une soirée passée avec telle vedette, tel artiste, dans tel loft underground, et ainsi de suite. Cette nouvelle «économie de l'expérience» $(E M, 276)$ impose un marché des désirs où les êtres consomment l'événement, se consomment et sont

$$
++
$$

31 Elfriede Jelinek, Drames de princesses. La jeune fille et la mort I-V, traduit de l'allemand par Magali Jourdan et Mathilde Sobottke, Paris, L'Arche, 2006 [2003], 138 p. 
consommés par autrui. Cette fabrique de soi ainsi que sa diffusion en images vident les êtres de toute intériorité. À la fin du troisième tableau, le personnage Quatre admet ce sentiment d'imposture :

\author{
QUATRE: Mais on dirait que depuis toujours \\ Je fais semblant d'être quelqu'un d'autre \\ Peut-être parce que je veux \\ Oui \\ Parce que je veux que vous m'aimiez \\ Mais là j'en peux plus \\ J'ai envie d'être moi-même une fois pour toutes. (NVN, 76)
}

Mais c'est pour mieux enchaîner (non sans humour) au tableau suivant sur la mise en scène de sa banalité quotidienne:

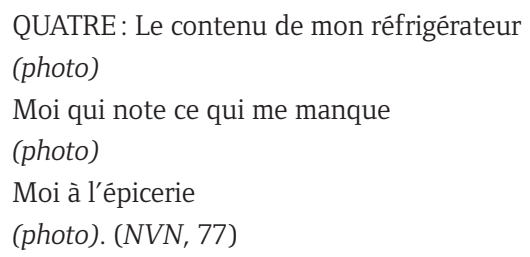

Dans la suite de la pièce, chaque personnage met en image la banalité de son existence, comme si cela était d'intérêt public: "Un kyste sur mon coude», «Moi qui me crève un bouton.» $(N V N, 84)$ Mais ces albums numériques d'images de soi insipides vont progressivement dégénérer en images sanglantes et sordides. Les récits adoptent alors une tonalité violente et autodestructrice, la surenchère spectaculaire opérant cette fois en sens inverse: chacun raconte sa descente aux enfers (réelle ou fantasmée), révélant de la sorte la face cachée du brillant spectacle de soi initial, lequel se terminera sur le suicide puis l'enterrement d'Un. Seront alors évoqués, sous forme de liste, les noms d'artistes qui se sont suicidés : Marilyn Monroe, Dédé Fortin, Kurt Cobain, Nelly Arcan, etc., autant de figures tragiques victimes du régime de la société de l'hyperspectacle, à laquelle leur existence était intimement chevillée. Mais même la mort d'Un devient un hyperévénement qui sera récupéré par le groupe d'amis pour être soumis à une sorte de vente aux enchères médiatique, laquelle déréalise la disparition d'un être à force de la «stariser»:

CINQ : J'ai imprimé ta photo sur des macarons

(Photo de photos)

J'en ai distribué des dizaines de milliers

Photo de photo

TROIS : J'ai imprimé ta photo en format géant

(Photo de photos)

Je la placarde partout dans la ville

Photo de vidéo de photos 
Revenons un moment à Debord, qui avait déjà analysé comment «le spectacle n'est pas un ensemble d'images, mais un rapport social entre des personnes médiatisées par des images ${ }^{32}$ ». Le spectacle est plus que jamais devenu une pratique sociale qui se présente comme un dédoublement du réel. Il s'oppose au réel en le représentant, tout en existant comme système concret et tangible. À l'inverse, le réel est usurpé par le spectacle, non parce que le spectateur le contemple, comme l'affirmait Debord, mais parce que l'individu a moulé son existence sur le monde écranique spectaculaire, ainsi que l'affirment Lipovetsky et Serroy. Il n'existe plus que par et dans les images et l'hyperspectacle de soi, lequel mêle réalité et vision fantasmée. Et Camille Brunelle dans tout cela? Présence discrète mentionnée par les personnages au début de la pièce, elle porte en elle, disent-ils, une beauté intérieure, une sensibilité, une écoute de l'autre, une fragilité, bref une humanité. Elle représente, en fait, cette vie intérieure qui semble à jamais perdue pour les cinq personnages, qui l'ont liquidée dans le flot étourdissant des images. À la fin de la pièce, lorsqu'un personnage montre une photo de Camille Brunelle, Quatre demande : «C'est qui elle?» $(N V N, 105)$ Elle figure ce lieu de l'être, le visage de chacun qui s'est effacé pour être remplacé par des simulacres de soi (photo de photo de photo...).

Si le thêâtre d'Olivier Choinière opère une virulente et habile critique de la société du spectacle, la toute jeune dramaturgie de Guillaume Corbeil s'attaque à l'hyperspectacle de soi dans la société médiatisée. Tous les deux recourent à des formes thêâtrales qui relèvent du dispositif performatif, en ce qu'ils mettent en jeu, dans la forme même de leurs créations, les mécaniques aliénantes des machines médiatiques qu'ils critiquent. Le spectateur est amené à vivre de l'intérieur la logique des esthétiques marchandes, que ce soit sous la forme du simulacre (Félicité), de la répétition qui va jusqu'à l'exaspération (Chante avec moi) ou de la surenchère expérientielle, qui adopte la forme de listes (Nous voir nous). Les univers fictionnels servent de cadre référentiel à un dispositif critique qui s'impose comme expérience concrète pour le lecteur/spectateur. Il est intéressant de noter, à cet égard, que la pièce de Corbeil prévoit aussi son propre dispositif scénique, lequel nécessite la projection de toutes les photos et vidéos mentionnées pour être pleinement opératoire. De la sorte, ces écritures inventent de nouvelles façons, moins narratives que performatives, $d$ 'interroger le rapport que nous entretenons à ce monde hypermédiatisé qui nous tient en otage.

\section{$+++$}

